\title{
Auto-Calibration and Motion Recovery from Silhouettes for Turntable Sequences
}

\author{
Hui Zhang, Guoqiang Zhang and Kwan-Yee Kenneth Wong* \\ Department of Computer Science, \\ The University of Hong Kong, \\ Pokfulam Rd, Hong Kong \\ \{hzhang, gqzhang, kykwong\}ecs.hku.hk
}

\begin{abstract}
This paper addresses the problem of structure and motion from silhouettes for turntable sequences. Previous works have exploited corresponding points induced by epipolar tangencies to estimate the image invariants under turntable motion and recover the epipolar geometry. In these approaches, however, camera intrinsics are needed in order to obtain Euclidean motion and reconstruction. This paper proposes a novel approach to precisely estimate the image invariants and the rotation angles in the absence of the camera intrinsics, and to perform auto-calibration. By exploiting a special parameterization of the epipoles, it is shown that the imaged circular points can be formulated in terms of the image invariants. A fixed scalar $\kappa$, introduced to account for the different scales in the homogeneous representations of the image invariants used in the parameterizations, is found crucial in both calibration and motion estimation. Given the image invariants, namely the horizon, the imaged rotation axis and its orthogonal vanishing point, this scalar can be determined from the epipoles in an image triplet. A robust method for estimating $\kappa$ is proposed and the rotation angles can be recovered using this estimated value of $\kappa$. All the estimated variables are then refined using bundle-adjustment and auto-calibration is performed using the imaged circular points, the imaged rotation axis and the associated vanishing point. This allows the recovery of the full camera positions and orientations, and hence Euclidean reconstruction. Experimental results demonstrate the simplicity of this novel approach and the high precision in the estimated motion and reconstruction.
\end{abstract}

\section{Introduction}

Turntable motion refers to the situation where the relative motion between a scene and a camera can be described as a rotation about a fixed axis. It has been widely used by computer vision and graphics researchers for generating 3D models. The most important and

\footnotetext{
*This project is supported by a grant from the Research Grants Council of the Hong Kong Special Administrative Region, China, under Project HKU 7155/03E.
} 
difficult step in the modelling process is to recover the relative pose of the camera and the rotation angles. Traditional approaches accomplish this task by careful calibration [15][10]. In [14], Fitzgibbon et al. introduced a point-based method to handle uncalibrated camera with unknown rotation angles. Their method is based on the projective geometry of the single axis motion, and it involves the computation of fundamental matrices and trifocal tensors. Jiang et al. [1][2] further extended this approach by making use of conic trajectories, and developed an algorithm that requires neither the computation of the fundamental matrices nor the trifocal tensors. However, all the above approaches require point correspondences and would therefore be unfeasible if the scene surfaces are smooth and textureless. In this situation, silhouettes become the only predominant and stable image feature. Approaches based on silhouettes generally exploit epipolar tangencies [3][9] to induce correspondences. However, such approaches require at least 7 epipolar tangencies and involve a nonlinear optimization with non-trivial initialization. Wong et al. [6] proposed a method based on only 2 epipolar tangencies between image pairs. However, this approach still involves a high-dimensional optimization and requires the knowledge of the camera intrinsics. Mendonça et al. [13] suggested that the epipolar tangencies can be obtained by an optimization based on a one-dimensional search. Nonetheless, the recovery of the rotation angles and furthermore the Euclidean reconstruction still require the knowledge of the camera intrinsics.

In this paper, we propose a novel approach to recover the rotation angles and camera intrinsics from silhouettes for turntable motion. Based on a special parameterization of the epipoles, we derive a formulation for the pair of imaged circular points in terms of the image invariants. Moreover, a fixed scalar, introduced to account for the different scales in the homogeneous representations of the image invariants used in the parameterizations, is found to be important and can be easily obtained from triples of images. A robust method for estimating this scalar is introduced, and the rotation angles between each pair of images can be easily and precisely recovered using this estimated scalar value. Autocalibration is then performed using the estimated circular points, the imaged rotation axis and the vanishing point. The estimated camera intrinsics then allows the recovery of the full camera positions and orientations, and hence a Euclidean reconstruction.

This paper is organized as follows. $\S 2$ reviews the recovery of the image invariants. $\S 3$ presents the recovery of the fixed scalar and the relative rotation angles. $\S 4$ proposes a novel formulation for the imaged circular points based on the image invariants and the fixed scalar. The camera calibration matrix is thus obtainable. $\S 5$ presents the algorithm and the implementations. $\S 6$ shows the experimental results and $\S 7$ gives the conclusions.

\section{Image Invariants under Turntable Motion}

Consider a reference camera $\mathbf{C}_{1}$ lying on the negative $Z$-axis of the world coordinate system, and rotating about the $Y$-axis (see Fig. 1(a)). The relative positions of the camera center describe a circle on a plane $\Pi_{h}$ orthogonal to the rotation axis. The image of $\Pi_{h}$ is the horizon (vanishing line) $\mathbf{l}_{h}$, whereas the image of the rotation axis is the line $\mathbf{l}_{s}$. Let the plane defined by the camera center and the rotation axis be $\Pi_{s}$, and we consider three orthogonal directions $N_{x}, N_{y}$ and $N_{z}$, given by the normal direction of $\Pi_{s}$, the $Y$-axis, and $N_{x} \times N_{y}$, respectively. These three directions will have vanishing points $\mathbf{v}_{x}, \mathbf{v}_{y}$ and $\mathbf{v}_{z}$, respectively. Note that $\mathbf{l}_{s}$ is also the image of $\Pi_{s}$ and hence $\mathbf{v}_{x}$ and $\mathbf{l}_{s}$ form a pole-polar 
relationship with respect to the image of the absolute conic [7]. By construction, $N_{x}$ is parallel to $\Pi_{h}$, and $N_{z}$ is parallel to both $\Pi_{h}$ and $\Pi_{s}$. Hence, $\mathbf{v}_{x}$ must lie on $\mathbf{l}_{h}$ and $\mathbf{v}_{z}$ is given by the intersection of $\mathbf{l}_{h}$ and $\mathbf{l}_{s}$. The pair of imaged circular points $\mathbf{i}$ and $\mathbf{j}$ must also lie on $\mathbf{l}_{h}$, hence $\mathbf{l}_{h}=\mathbf{i} \times \mathbf{j}$. If the intrinsic parameters of the camera is kept constant, then all the aforementioned image entities will be fixed (see Fig. 1(b)).

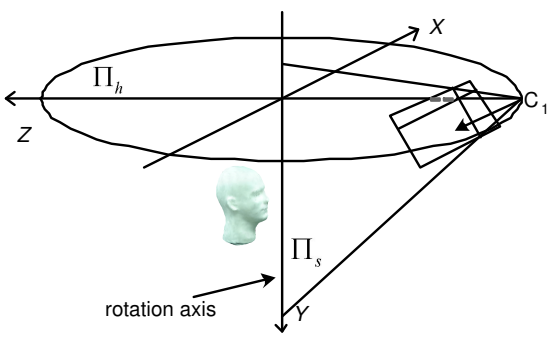

(a)

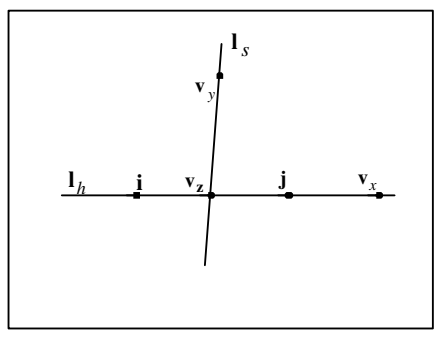

(b)

Figure 1: Turntable motion.(a)3D geometry. (b)2D Image invariants.

In [13], it has been shown that $\mathbf{l}_{s}$ and $\mathbf{v}_{x}$ can be recovered by exploiting the symmetry property exhibited in the image of the surface of revolution (SOR) swept out by the rotating object. Images in a dense sequence are overlapped to obtain the image profile of the virtual SOR which is invariant to a harmonic homology [11][13], given by

$$
\mathbf{W}=\mathbf{I}-2 \frac{\mathbf{v}_{x} \mathbf{I}_{s}^{\mathbf{T}}}{\mathbf{l}_{x}^{\mathbf{T}} \mathbf{l}_{s}}
$$

Initial estimates for $\mathbf{l}_{s}$ and $\mathbf{v}_{x}$ can then be obtained by locating bi-tangent points in the profile, and are further refined by minimizing the transformation error introduced by $\mathbf{W}$ (see [13] for details).

The horizon $\mathbf{l}_{h}$ can be estimated as the line robustly fitted to the epipoles (i.e., the images of the camera center) between all image pairs and passing through $\mathbf{v}_{x}$. To obtain an initial set of epipoles, epipolar tangents have to be recovered first. This requires finding a line tangent to one silhouette which is transformed by $\mathbf{W}^{-\mathbf{T}}$ to a line tangent to the silhouette in the other image (see Fig. 2). The search for corresponding tangents may be carried out as a one dimensional optimization problem by minimizing the distance from the transformed line $\mathbf{l}_{i}^{\prime}=\mathbf{W}^{-\mathbf{T}} \mathbf{l}_{i}$ to the profile in the second image. The single parameter is the angle that defines the orientation of the epipolar line $\mathbf{l}_{i}$ in the first image [13]. The epipoles can then be computed as the intersection of epipolar lines in the same image.

Note that under turntable motion, the fundamental matrix relating any two views can be explicitly parameterized in terms of the image invariants, and is given by [14][13]

$$
\mathbf{F}(\theta)=\left[\mathbf{v}_{x}\right]_{\times}+\kappa \tan \frac{\theta}{2}\left(\mathbf{l}_{s} \mathbf{l}_{h}^{\mathbf{T}}+\mathbf{l}_{h} \mathbf{l}_{s}^{\mathbf{T}}\right)
$$

where $\theta$ is the rotation angle between the two views. The terms in (2) are in homogeneous coordinates, and the scalar $\kappa$ is therefore necessary to account for the different scales used in the representations. The scalar $\kappa$ is unknown but fixed for any angle $\theta$, and cannot be obtained from two images alone. Note that in (2), the only variable is the rotation angle $\theta$. It will be shown in $\S 4$ that the imaged circular points can be parameterized in terms of the remaining fixed entities (i.e., $\mathbf{v}_{x}, \mathbf{l}_{s}, \mathbf{l}_{h}$ and $\kappa$ ). 


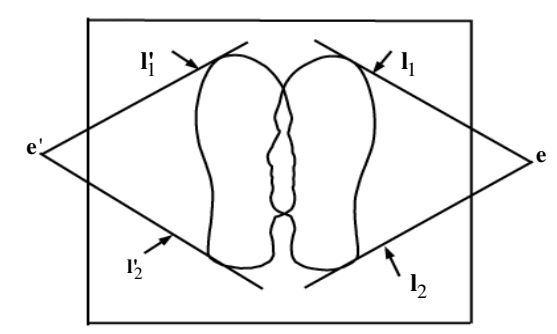

Figure 2: The overlapping of two silhouettes and their epipolar tangents. $\mathbf{l}_{1}, \mathbf{l}_{1}^{\prime}, \mathbf{l}_{2}$ and $\mathbf{l}_{2}^{\prime}$ are the outer epipolar tangent lines.

\section{Recovery of the Fixed Scalar and the Rotation Angles}

Using the parameterization in (2), the epipoles $\mathbf{e}_{i}(i=1,2)$ in an image pair, obtained from the left and right nullspaces of the fundamental matrix, are given by [13]

$$
\mathbf{e}_{i} \sim \mathbf{v}_{x}-(-1)^{i} \kappa \tan \frac{\theta}{2} \mathbf{l}_{s} \times \mathbf{l}_{h}
$$

Given the estimated $\mathbf{l}_{s}, \mathbf{v}_{x}$ and $\mathbf{l}_{h}$ (see $\S 2$ ), the only missing term in the parameterization of the epipole is the coefficient $\kappa \tan \frac{\theta}{2}$. This parameter can be found by a one-dimensional search that minimizes the distance between the transformed epipolar lines and the corresponding tangent points [13].

Consider a triplet of views with relative rotation angles $\theta_{p q}(p, q=1,2,3 p \neq q)$ (see Fig. 3(b), which only shows the variables relating views 1 and 2 , and 2 and 3). Let $\gamma_{1}=\kappa \tan \frac{\theta_{12}}{2}, \gamma_{2}=\kappa \tan \frac{\theta_{23}}{2}$, and $\gamma_{3}=\kappa \tan \frac{\theta_{13}}{2}$. Using the fact that $\theta_{13}=\theta_{12}+\theta_{23}$, the fixed scalar $\kappa$ can be easily obtained as

$$
\kappa=\sqrt{\frac{\gamma_{1} \gamma_{2} \gamma_{3}}{\gamma_{3}-\gamma_{1}-\gamma_{2}}}
$$

The rotation angles between each pair of images can then be directly obtained by dividing $\gamma$ by $\kappa$.

\section{Recovery of the imaged circular points and intrinsics}

In this section, a new parameterization for the imaged circular points in terms of the image invariants under turntable motion and the fixed scalar $\kappa$ (introduced in $\S 2$ ) is presented. Such a parameterization allows the imaged circular points to be recovered directly from those estimated fixed entities and provides constraints for auto-calibration.

Theorem I. Given the vanishing point $\mathbf{v}_{x}$, the image $\mathbf{l}_{s}$ of the rotation axis and the horizon $\mathbf{l}_{h}$ of the turntable plane, the pair of circular points $\mathbf{i}, \mathbf{j}$ of the turntable plane are given by

$$
\mathbf{i}, \mathbf{j} \sim \mathbf{v}_{x} \pm j \kappa \mathbf{l}_{s} \times \mathbf{l}_{h},
$$

where $j^{2}=-1, \kappa$ is the fixed scalar in (2), and the left and right hand sides of $\sim$ are equal up to a scalar. 


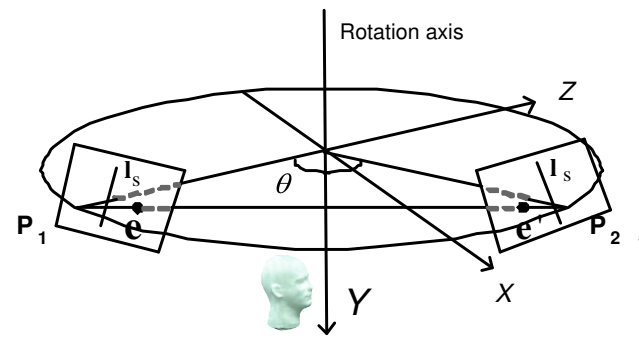

(a)

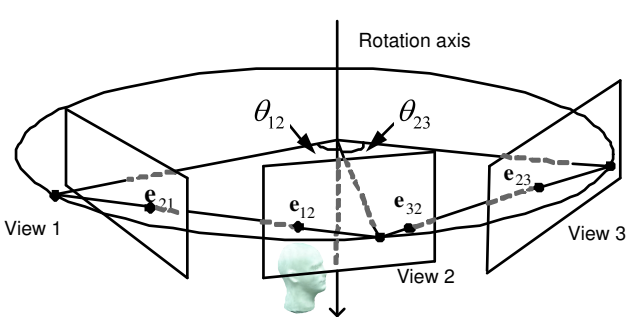

(b)

Figure 3: (a)A pair of cameras $\mathbf{P}_{\mathbf{1}}, \mathbf{P}_{\mathbf{2}}$ are related by a rotation with an angle $\theta$ about the $Y$-axis. (b)Three views under turntable motion. Epipole $\mathbf{e}_{p q}$ is the projection of the camera center of view $p$ onto view $q$. $\theta_{p q}$ is the rotation angle between view $p$ and view $q(p, q=1,2,3 p \neq q)$.

Proof. Consider a pair of cameras $\mathbf{P}_{1}=\mathbf{K R}[\mathbf{I} \mid-\mathbf{C}]$ and $\mathbf{P}_{2}=\mathbf{K R}\left[\mathbf{R}_{Y}(\theta) \mid-\mathbf{C}\right]$, where $\mathbf{R}_{Y}(\boldsymbol{\theta})$ is a rotation about the $Y$-axis by an angle $\theta$ and $\mathbf{C}=[00-1]^{\mathbf{T}}$ is the camera center (see Fig. 3(a)). The image $\mathbf{x}$ of a point $\mathbf{X}=\left[\begin{array}{lll}X & 0 Z 1\end{array}\right]^{\mathbf{T}}$ on the $X-Z$ plane under $\mathbf{P}_{1}$ is given by

$$
\mathbf{x} \sim\left[\begin{array}{llll}
\mathbf{p}_{1} & \mathbf{p}_{2} & \mathbf{p}_{3} & \mathbf{p}_{3}
\end{array}\right]\left[\begin{array}{c}
X \\
0 \\
Z \\
1
\end{array}\right]=\left[\begin{array}{ll}
\mathbf{p}_{1} & \mathbf{p}_{3}
\end{array}\right]\left[\begin{array}{c}
X \\
Z+1
\end{array}\right],
$$

where $\mathbf{p}_{c}(c=1,2,3)$ are the columns of $\mathbf{P}_{1}$. It can be easily seen that $\mathbf{p}_{1} \sim \mathbf{v}_{x}, \mathbf{p}_{3} \sim \mathbf{v}_{z} \sim$ $\mathbf{l}_{s} \times \mathbf{l}_{h}[14]$. The epipole $\mathbf{e}_{i}$ is the projection of $\left[\sin \left(\pi+(-1)^{i} \theta\right) 0 \cos \left(\pi+(-1)^{i} \theta\right) 1\right]^{\mathbf{T}}$ on the image of camera $\mathbf{P}_{i}$, and is given by

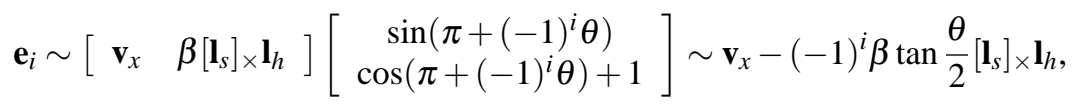

where $\beta$ is an unknown fixed scalar used to fix the relative scale between $\mathbf{v}_{x}$ and $\mathbf{l}_{s} \times \mathbf{l}_{h}$. By comparing (3) and (7), it can be easily derived that $\beta=\kappa$. Note $\mathbf{i}, \mathbf{j}$ are the image of the circular points $\mathbf{I}, \mathbf{J}=[10 \pm j 0]^{\mathbf{T}}$, i.e.,

$$
\mathbf{i}, \mathbf{j} \sim \mathbf{p}_{1} \pm j \mathbf{p}_{3}
$$

By putting the corresponding terms into (8), the theorem is proved.

The obtained images of the circular points can be used to find the camera intrinsics. As they lie on the image of the absolute conic (IAC) $\omega$, it results in the following two constraint:

$$
\mathbf{i}^{\mathbf{T}} \omega \mathbf{i}=0 \text { and } \mathbf{j}^{\mathbf{T}} \omega \mathbf{j}=0 .
$$

Besides, the imaged rotation axis $\mathbf{l}_{s}$ and the vanishing point $\mathbf{v}_{x}$ define a pole-polar relationship w.r.t. the IAC [11], i.e.,

$$
\mathbf{l}=\omega \mathbf{v}
$$

and this provides one additional independent constraints on $\omega$. By Cholesky decomposition [4] of $\omega$, a natural camera with zero skew and unit aspect ratio can be calibrated. 


\section{Implementation}

A dense image sequence is used here to generate the image of the virtual SOR swept out by the rotating object, and good estimates for $\mathbf{l}_{s}$ and $\mathbf{v}_{x}$ can be obtained by exploiting the symmetry property of this image. A sub-sequence is then used for a precise recovery of the motion and the camera intrinsics. Cubic B-spline snake [8] is used to extract silhouettes from the image sequence since it can achieve sub-pixel localization accuracy. Besides, it also facilitates the search of epipolar tangents. An initial set of epipoles from some randomly sampled image pairs in the sub-sequence is obtained, and the horizon $\mathbf{l}_{h}$ is then recovered by RANSAC.

For successive image pairs, an initial set of epipoles is calculated using (3) where the coefficient $\kappa \tan (\theta / 2)$ is found by an one-dimensional search that minimizes the distance between the epipolar lines transformed by $\mathbf{W}^{-\mathbf{T}}$ and the corresponding tangent points [13]. The fixed scalar $\kappa$ can then be recovered from successive image triplets by (4). However, due to the existence of noises, the values of $\kappa$ estimated from different triplets may have different values. A robust method is therefore proposed to estimate $\kappa$. A set of $\kappa$ is recovered from some randomly sampled image triplets and the mode $\kappa_{0}$ is then selected as the best estimate. The initial values of the rotation angles can be precisely obtained by dividing the coefficient $\kappa \tan (\theta / 2)$ by $\kappa_{0}$.

However, in practice, a few angles may be poorly estimated and a refinement is needed. The error angles can be identified by the ratio between the estimated $\kappa$ and $\kappa_{0}$. The angle between view $p$ and view $p+1$ can be refined by using eight adjacent view triplets, i.e., $(p-4, p, p+1),(p-3, p, p+1),(p-2, p, p+1),(p-1, p, p+1)$, $(p, p+1, p+2),(p, p+1, p+3),(p, p+1, p+4),(p, p+1, p+5)$, and the best angle can be identified by the smallest difference between the estimated $\kappa$ and $\kappa_{0}$.

Finally, a bundle-adjustment using Levenberg Marquardt minimization [5] could be applied to refine all the parameters, i.e., $\mathbf{l}_{s}, \mathbf{l}_{h}, \mathbf{v}_{x}, \kappa$ and the $n-1$ rotation angles for a sequence of $n$ images. As $\mathbf{l}_{h}^{\mathbf{T}} \mathbf{v}_{x}=0,5+n$ parameters are to be refined. The intrinsics are then estimated with the optimized entities, followed by a Euclidean reconstruction. Algorithm 1 summarized the complete procedures of the technique.

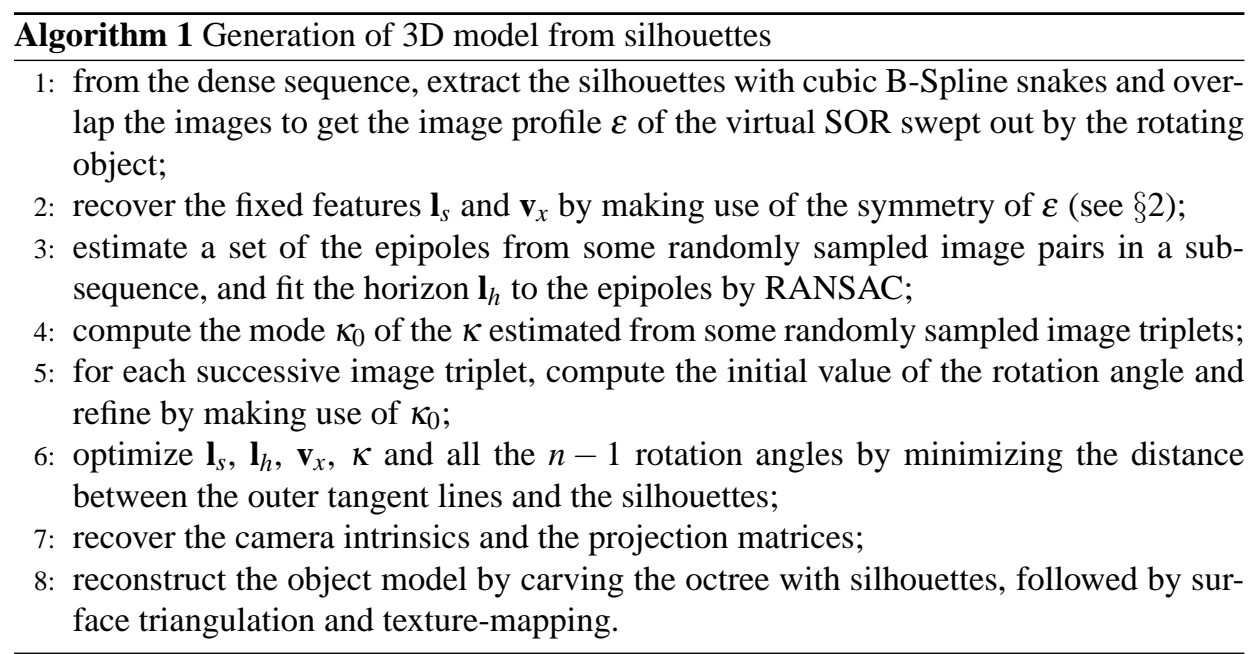




\section{Experimental Results}

Experiments on real turntable sequences were carried out to test the feasibility of the proposed approach. The first sequence consisted 72 images of a vase (see Fig. 4). The image had a resolution of $640 \times 480$. Each image was taken by sequentially rotating the object by $5^{\circ}$ on a manually operated turntable with a resolution of $0.01^{\circ}$. After obtaining $\mathbf{l}_{s}, \mathbf{v}_{x}$ and $\mathbf{l}_{h}$ from this dense sequence, a sub-sequence consisting of 18 images with successive rotation angles of $20^{\circ}$ was extracted for the auto-calibration and motion estimation. Fig. 5 (a) shows the histogram of the fixed factor $\kappa$ estimated from some randomly sampled image triplets in the sub-sequence, and the peak indicates the best $\kappa_{0}$. Fig. 6(a) shows the ratios of the initially estimated $\kappa$ s using a single triplet of successive images to the best estimate $\kappa_{0}$. Fig. 6(b) shows both the initially recovered angles and the refined angles. It can be seen that the initial angles already had a good precision while the refined angles were even better, with a rms error of only $0.174^{\circ}$. These angles were further refined using the bundle-adjustment optimization and a rms error of $0.123^{\circ}$ was achieved, which is better than the rms error of $0.2^{\circ}$ for the same vase sequence in [13]. Under the assumption of a natural camera (zero-skew and unit aspect ratio), table 1 compares the auto-calibration result with that of the ground-truth, which was obtained with the classical L-shape grid pattern [12]. It can be seen that the focal length $f$ and the $\mathbf{u}_{0}$ coordinate of the principal point were both precisely estimated while $\mathbf{v}_{0}$ was not. This is due to the error in the estimated $\mathbf{v}_{x}$. Fig. 7 shows three views of the $3 \mathrm{D}$ model reconstructed from the estimated motion.

Similarly, the second sequence consists of 72 images of a head model with successive rotation angles of $5^{\circ}$ (see Fig. 8). The electronic turntable used has a resolution of $0.2^{\circ}$. After obtaining $\mathbf{l}_{s}, \mathbf{v}_{x}$ and $\mathbf{l}_{h}$ from this dense sequence, a sub-sequence consisting of 18 images with successive rotation angles of $20^{\circ}$ was extracted. Fig. 5(b) shows the histogram of the fixed factor $\kappa$ estimated. Fig. 9 (a) shows the ratios of the initially estimated values for $\kappa$ using triplet of images to the best estimate $\kappa_{0}$. Fig. 9 (b) shows the initially recovered angles, the refined angles (with a rms error of $0.233^{\circ}$ ), and those obtained by bundle-adjustment (with a rms error of $0.171^{\circ}$ ). The reconstructed model is shown in Fig. 10 , which reflects good qualities of our estimated parameters.
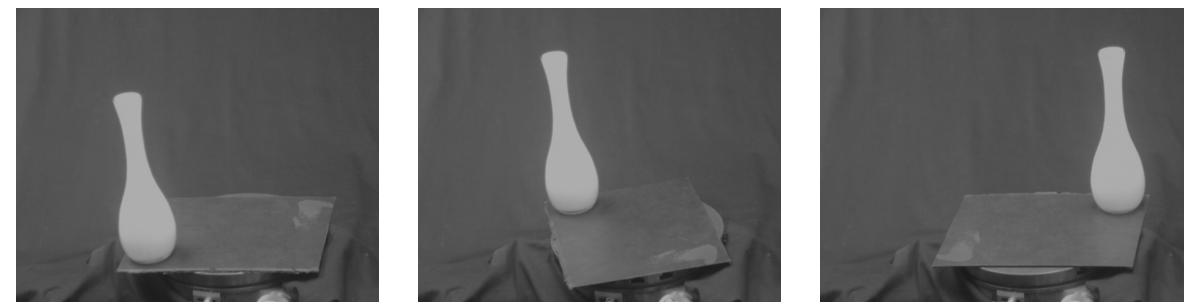

Figure 4: An image sequence of a vase under turntable motion.

\section{Conclusions}

In this paper, we have presented a simple and practical approach for recovering the camera intrinsics and the relative angles using silhouettes under turntable motion. The system 


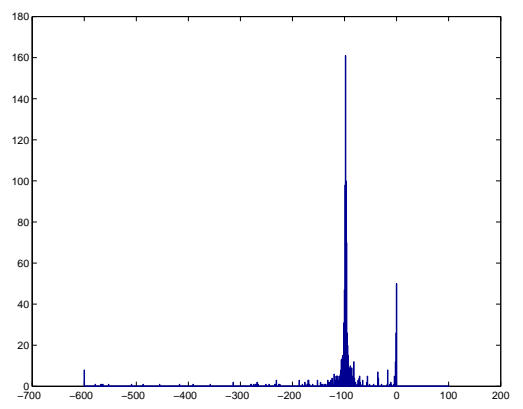

(a)

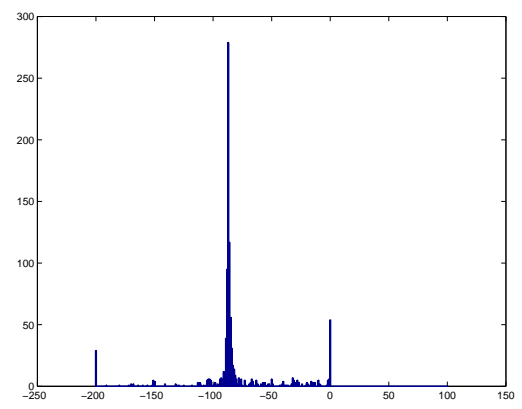

(b)

Figure 5: Histogram of the estimated values for $\kappa$, and the mode is chosen as the "best estimate". (a) For the vase sequence. (b) For the head sequence.

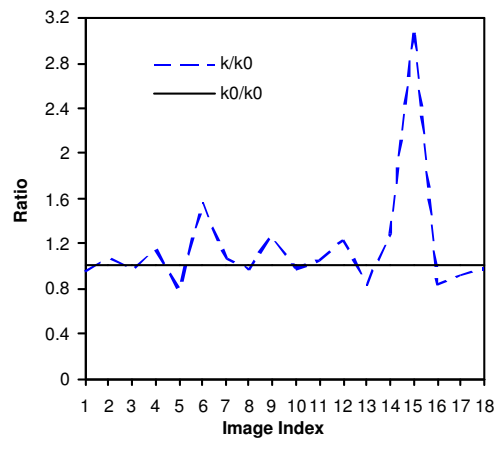

(a)

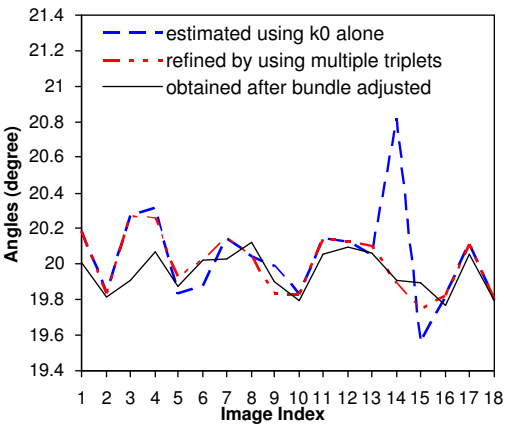

(b)

Figure 6: Vase sequence. (a) The ratio of initially estimated $\kappa \mathrm{s}$ to $\kappa_{0}$. The larger the difference, the worse the $\kappa$. (b) Angles estimated using $\kappa_{0}$ alone, which are refined by using multiple triples and finally obtained after bundle adjustment.

input is only uncalibrated images of a dense turntable sequence. We have proposed a simple formulation of the imaged circular points in terms of the image invariants and the fixed scalar. The fixed scalar can be robustly estimated and used for angle estimation and refinement. The rotation angles can therefore be estimated with a high precision without the knowledge of camera intrinsics. The system only involves some simple minimization procedures. Auto-calibration has been carried out in our work to achieve Euclidean reconstruction. Experiments on two image sequences have produced convincing 3D models, demonstrating the practicality of our algorithm.

\section{References}

[1] Jiang G., Tsui H.T., Quan L., and Zisserman A. Single axis geometry by fitting conics. In European Conf. on Computer Vision, pages 482-488, 2002.

[2] Jiang G., Quan L., and Tsui H.T. Circular motion geometry by minimal 2 points in 4 images. In Proc. ICCV, pages 221-227, 2003. 


\begin{tabular}{c|ccc}
\hline- & $\mathrm{f}$ & $u_{0}$ & $v_{0}$ \\
\hline ground-truth & 2389.8 & 342.83 & 255.32 \\
estimated value & 2370.7 & 323.81 & 317.13 \\
percentage error & $0.80 \%$ & $5.55 \%$ & $24.21 \%$ \\
\hline
\end{tabular}

Table 1: Estimated and ground-truth values for intrinsics of vase sequence.
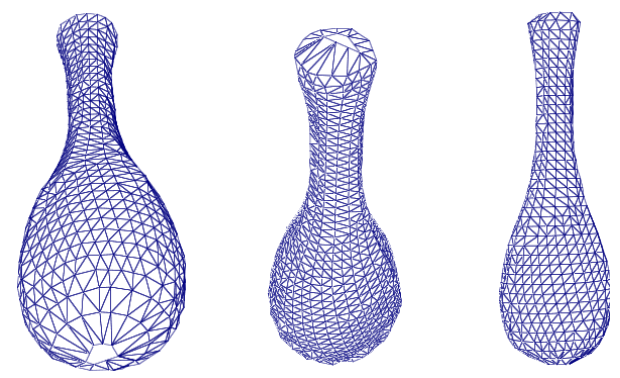

Figure 7: 3D model of the vase built from the estimated turntable motion.

[3] Porrill J. and Pollard S. B. Curve matching and setreo calibratoin. Image and Vision Computing, 9(1):45-50, 2004.

[4] Gentle J.E. Numerical Linear Algebra for Applications in Statistics. Springer-Verlag, 1998.

[5] More J.J. The levenberg-marquardt algorithm: Implementation and theory. In Numerical Analysis, Lecture Notes in Mathematics 630, pages 105-116, 1977.

[6] Wong K.-Y. K. and Cipolla R. Structure and motion from silhouettes. In Proc. 8th IEEE ICCV, volume II, pages 217-222, Vancouver, Canada, July 2001.

[7] Faugeras O.D., Luong Q.-T., and Maybank S.J. Camera self-calibration: Theory and experiments. In ECCV92, pages 321-334, 1992.

[8] Cipolla R. and Blake A. Surface shape from the deformation of apparent contours. Int. Journal of Computer Vision, 9(2):83-112, November 1992.

[9] Cipolla R., Aström K., and Giblin P. Motion from the frontier of curved surfaces. In Proc. 5th ICCV, pages 269-275, Jun 1995.

[10] Szeliki R. Shape from rotation. In Proc. of IEEE CVPR, pages 625-630, 1991.

[11] Hartley R.I. and Zisserman A. Multiple View Geometry in Computer Vision. Cambridge University Press, Cambridge, UK, 2000.

[12] Tsai R.Y. A versatile camera calibration technique for high accuracy $3 \mathrm{~d}$ machine vision metrology using off-the-shelf tv cameras and lenses. IEEE Journal of Robotics and Automation, 3(4):323-344, 1987.

[13] Mendonça P. R. S., Wong K.-Y. K., and Cipolla R. Camera pose estimation and reconstruction from image profiles under circular motion. In Proc. ECCV, volume II, pages 864-877, 2000.

[14] Fitzgibbon A. W., Cross G., and Zisserman A. Automatic 3d model construction for turn-table sequences. In 3D Structure from Multiple Images of Large-Scale Environments, LNCS 1506, pages 155-170. Springer Verlag, June 1998.

[15] Niem W. Robust and fast modelling of $3 \mathrm{~d}$ natural objects from multiple views. In Proc. SPIL, volume Vol. 2182, pages 388-397, 1994. 

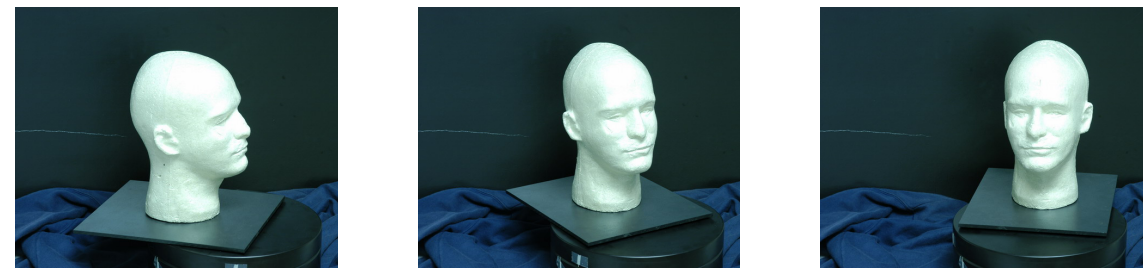

Figure 8: An image sequence of a polystyrene head model under turntable motion.

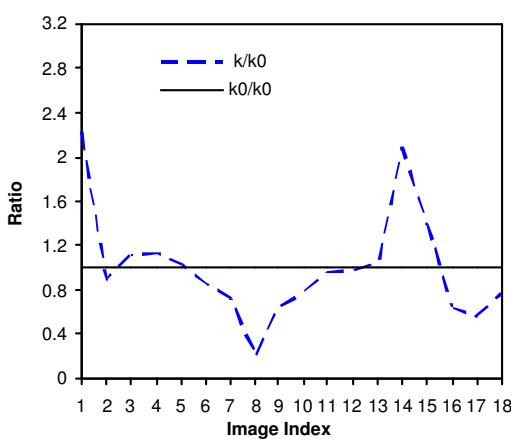

(a)

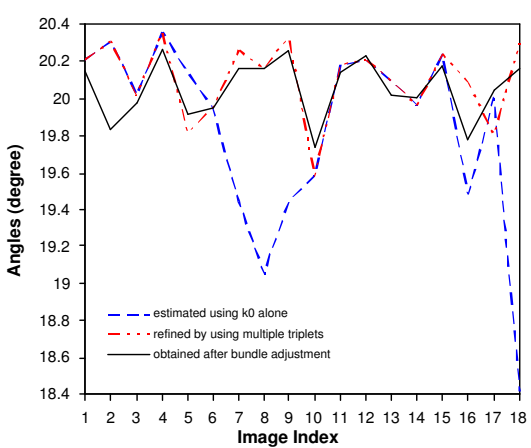

(b)

Figure 9: Head sequence. (a) The ratio of initially estimated $\kappa \mathrm{s}$ to $\kappa_{0}$. The larger the difference, the worse the $\kappa$. (b) Angles estimated using $\kappa_{0}$ alone, which are refined by using multiple triples and finally obtained after bundle adjustment.
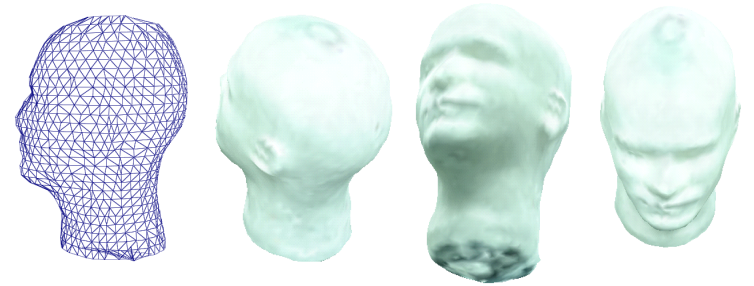

Figure 10: 3D model of the polystyrene head built from the estimated turntable motion. 